\title{
Different Causes of Pyrexia of Unknown Origin on Bone Marrow Examination- An Institutional Experience
}

\author{
Ritu Bhagat ${ }^{1}$, Roopali Jandial2, Vinod Kumar ${ }^{3}$ \\ ${ }^{1}$ Department of Pathology, GMC, Jammu, Jammu and Kashmir, India. ${ }^{2}$ Department of Pathology, GMC, Jammu, \\ Jammu and Kashmir, India. ${ }^{3}$ Department of Medicine, GMC, Jammu, Jammu and Kashmir, India.
}

\section{ABSTRACT}

\section{BACKGROUND}

Petersdorf and Beeson defined pyrexia of unknown origin (PUO) as a complaint with temperature surpassing $38.3^{\circ} \mathrm{C}$, developing over a period of at least three weeks, with no possible opinion reached after one week of inpatient investigation. In the present study, an attempt has been made to find out the causes of PUO based on bone marrow morphology. The range of diseases causing PUO not only seems to be determined by geographical factors, but time also plays a vital role. Bone marrow examination plays an important role in early diagnosis of core cause for PUO and is the best tool for picking haematological and non-haematological disorders in any age group.

\section{METHODS}

All patients presenting with classical PUO coming to Government Medical College, Jammu, fulfilling the criteria of Petersdorf RG et al whether inpatient or outpatient over a period of two years were included in this cross-sectional study.

\section{RESULTS}

Out of 76 patients, 48 were males and 28 were females. Age of patients varied from 12 years to 70 years. Majority of patients were in the age group of 30-44 years comprising of $45 \%$ of total cases. Anaemia was seen in nearly $50 \%$ of cases of PUO. Most common diagnosis was neoplastic changes, seen in $20 \%$ of patients, $16 \%$ cases show megaloblastic changes, iron deficiency was seen $10 \%$ cases, reactive myeloid hyperplasia was seen in $18 \%$ cases, haemophagocytosis in $6 \%$ cases, $5 \%$ cases showed hypocellular marrow. Among infections, malaria was the commonest constituting $5.2 \%$ cases. Out of total of 15 cases of neoplastic changes in bone marrow, majority of them were acute myeloid leukaemia seen in $40 \%$ cases.

\section{CONCLUSIONS}

Bone marrow examination is an important investigation of PUO in arriving at an etiological diagnosis. The most frequent causes of pyrexia of unknown origin observed in children were acute lymphoblastic leukaemia, megaloblastic anaemia and haemophagocytosis, whereas in adults, the main causes were malignancies, megaloblastic anaemia and reactive myeloid hyperplasia. This study sheds light on the current spectrum of diseases causing pyrexia of unknown origin in this region.

\section{KEY WORDS}

Pyrexia of Unknown Origin, Bone Marrow Examination, Malignancy, Haematological
Corresponding Author:

Dr. Vinod Kumar,

C/o Sri K. C. Bhagat, \#209 Colonel Colony,

Bohri-Talabtillo, Jammu-180002,

Jammu and Kashmir, India.

E-mail:drvkatil76@gmail.com

DOI: $10.14260 / j e m d s / 2020 / 111$

Financial or Other Competing Interests: None.

How to Cite This Article:

Bhagat R, Jandial R, Kumar V. Different causes of pyrexia of unknown origin on bone marrow examination- an institutional experience. J. Evolution Med. Dent. Sci. 2020;9(08):492-495, DOI: $10.14260 /$ jemds/2020/111

Submission 11-07-2019,

Peer Review 29-01-2020,

Acceptance 05-02-2020,

Published 24-02-2020.

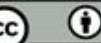




\section{BACKGROUND}

Petersdorf and Beeson defined pyrexia of unknown origin (PUO) as a complaint with temperature surpassing $38.3^{\circ} \mathrm{C}$, developing over a period of at least three weeks, with no possible opinion reached after one week of inpatient investigation. ${ }^{1}$ The range of diseases causing PUO not only seems to be determined by geographical factors, but time also plays a vital role. ${ }^{2}$ Bone marrow examination plays an important role in early diagnosis of core cause for PUO and is a best tool for picking haematological and non-haematological disorders in any age group. ${ }^{3,4}$

In developing countries affordability is a limitation; so bone marrow examination has become the ultimate modality for early diagnosis of pyrexia of unknown origin. Variable response of the bone marrow depends upon aetiology either infective or non-infective, as a result infections and systemic diseases can be studied by analysis of morphology \& aetiology and helps in the management of patients with PUO.

This cross sectional study is conducted to find out the spectrum of diseases causing PUO in Jammu region. In present study an attempt has been made to find out the causes of PUO based on bone marrow morphology.

\section{METHODS}

All the patients presenting with classical PUO (pyrexia of unknown origin) coming to Government Medical College, Jammu, fulfilling the criteria of Petersdorf RG et al, ${ }^{1}$ whether inpatient or outpatient over a period of two years were included in this cross sectional study. Detailed clinical history, physical examination and various relevant investigations were noted from the case records. Preliminary investigations include routine urine examination, haemogram, peripheral blood smear for malarial parasites, liver function tests, urea \& creatinine, Widal test, Mantoux test and chest $\mathrm{x}$ ray. Bone marrow aspiration had been done after taking an informed consent from the patient. Mostly posterior superior iliac spine was the site preferred under local anaesthesia using lumbar puncture needle of 16G. The aspirate smears were stained with Giemsa stain. The parameters studied were cellularity of marrow particles; status of all series of haematopoietic cells increase in plasma cells presence and type of granulomas and any other associated features seen including haem phagocytosis. Got approval from IEC and consent was taken from all patients.

\section{Statistical Analysis}

The Statistical Package for Social Science (SPSS) Version 20 will be used for Data Analysis. Mean, median, and SD are used to describe quantitative data. Qualitative data are summarized using frequency and percentage.

\section{RESULTS}

A total of 76 patients of PUO who underwent bone marrow aspiration over a period of two years were included in our study. Out of 76 patients 48 were males and 28 were females, with a M: F ratio of 1.7:1. Age of patients varied from 12 years to 70 years. Out of 76 patients, there were 56 adult patients and 09 children ( $<14$ years).Majority of patients were in the age group of $30-44$ years comprising of $45 \%$ of total cases followed by $15-29$ years, $33 \%$ cases. Age distribution is shown in table 1.

\begin{tabular}{|c|c|c|}
\hline Age Group (Years) & No. of Cases & Percentage (\%) \\
\hline $1-14$ & 09 & 12 \\
\hline $15-29$ & 25 & 33 \\
\hline $30-44$ & 34 & 45 \\
\hline $45-59$ & 05 & 06 \\
\hline$>60$ & 03 & 04 \\
\hline Total & $\mathbf{7 6}$ & $\mathbf{1 0 0}$ \\
\hline \multicolumn{2}{|c|}{ Table 1. Age Distribution of Patients with PUO } \\
\hline
\end{tabular}

All the patients gave history of prolonged fever ranging in duration from 20 days to 120 days, with an average duration of 30 days. There was history of weight loss in 35 cases, history of diarrhoea in 08 cases, epistaxis seen in 03 cases, history of rash and jaundice seen in 03 cases. Hepatosplenomegaly was seen in 30 cases and lymphadenopathy in 14 cases. Anaemia was seen in nearly $50 \%$ of cases of PUO. It was normocytic normochromic in $70 \%$ of cases, macrocytic in $16 \%$ cases and microcytic hypochromic in $14 \%$ cases.

Various morphological changes were seen in patients with PUO on bone marrow aspiration which are shown in table 2 . Most common diagnosis was neoplastic changes, seen in $20 \%$ of patients, $16 \%$ cases show megaloblastic changes, iron deficiency was seen $10 \%$ cases, reactive myeloid hyperplasia seen in $18 \%$ cases, haemophagocytosis in $6 \%$ cases, $5 \%$ cases show hypo cellular marrow. Normal marrow findings seen $5.3 \%$ cases. Among infections malaria was the commonest constituting $5.2 \%$ cases, intracellular and extracellular amastigote forms of leishmania donovani were seen in $4 \%$ cases and tuberculosis was seen in $2.6 \%$ cases. Also $2.6 \%$ cases show features of ITP.

\begin{tabular}{|c|c|c|}
\hline Diagnosis & No. of Cases & Percentage (\%) \\
\hline Normal marrow & 07 & 5.3 \\
\hline Neoplastic & 15 & 20 \\
\hline Megaloblastic & 12 & 16 \\
\hline Reactive myeloid hyperplasia & 14 & 18 \\
\hline Iron deficiency & 08 & 10 \\
\hline Haemophagocytosis & 05 & 06 \\
\hline Hypocellular marrow & 04 & 05 \\
\hline Tuberculosis & 02 & 2.6 \\
\hline Leishmaniasis & 03 & 04 \\
\hline Malaria & 04 & 5.2 \\
\hline ITP & 02 & 2.6 \\
\hline Table 2. Morphological Changes in Bone Marrow \\
\hline
\end{tabular}

Out of total 15 cases of neoplastic changes in bone marrow majority of them were acute myeloid leukemia seen in $40 \%$ cases. Acute lymphoid leukemia was the second common diagnosis constituting about $26.6 \%$ cases, all of them were seen in children. Chronic myeloid leukemia and multiple myeloma were seen in $13.3 \%$ cases each. Myeloid dysplastic syndrome was seen in $6.7 \%$ cases. Distribution of malignancy is shown in table 3.

\begin{tabular}{|c|c|c|}
\hline Malignancy & No. of Cases & Percentage (\%) \\
\hline Acute lymphoid leukemia & 04 & 26.6 \\
\hline Acute myeloid leukemia & 06 & 40 \\
\hline Chronic myeloid leukemia & 02 & 13.3 \\
\hline Multiple myeloma & 02 & 13.3 \\
\hline MDS & 01 & 6.7 \\
\hline Total & $\mathbf{1 5}$ & $\mathbf{1 0 0}$ \\
\hline \multicolumn{2}{|c}{ Table 3. Distribution of Malignancies } \\
\hline
\end{tabular}


Among children with PUO most common finding on bone marrow aspiration was acute lymphoid leukemia 04 cases, followed by 03 cases of megaloblastic anaemia and 02 cases of haemophagocytosis.

\section{DISCUSSION}

Comparison of patients with pyrexia of unknown origin is difficult because of the large number of possible causes and the influence of numerous factors on the various diagnostic categories. Literature review has shown that bone marrow studies should be considered significant in evaluating patients having long duration of pyrexial illness. ${ }^{5}$ Different infections, haematological and nonhematological malignancies are well differentiated on bone marrow examination, cultures and trephine biopsy results. ${ }^{6}$ Though bone marrow aspiration and trephine biopsy is a painful procedure but the diagnosis made by this can be lifesaving in many patients. 7,8

A variety of morphologic changes in the bone marrow have been described in various infectious and systemic diseases resulting in PUO. ${ }^{9}$ These changes may be features of acute inflammation (interstitial oedema, vascular congestion, haemorrhage, ischemic necrosis or suppurative necrosis) or chronic inflammation with granuloma formation, reactive lymphoid hyperplasia, plasmacytosis, histiocytosis or fibrosis. ${ }^{9}$

In our study, the various causes of PUO were neoplasm, megaloblastic anaemia, iron deficiency anaemia, reactive myeloid hyperplasia followed by haemophagocytosis, hypo cellular marrow, infections like tuberculosis, malaria and leishmania. This is in contrast to other studies where infections constituted the most common cause followed by neoplasm and collagen vascular disease. ${ }^{10,11,12}$ Another study conducted by Elisabeth et al and Netherlands about FUO in 167 patients, showed infection as a leading cause $(26 \%)$ followed by neoplasm and non-infectious inflammatory disease $13 \%$ \& $24 \%$ respectively). Miscellaneous causes accounted 5\% and $30 \%$ of cases were undiagnosed despite every effort.

In our study $20 \%$ cases showed haematological malignancies in their bone marrow. Most common neoplasm among them was acute lymphoid leukemia, 04 cases $(26.6 \%)$ all cases were seen in children. Second common was Acute myeloid leukemia 06 cases (40\%) followed by Chronic myeloid leukemia and multiple myeloma 02 cases (13.3\%) each and Myeloid dysplastic syndrome constituted only 01 case $(6.7 \%)$ as shown in table 3 . These findings are similar to study done by Haq SA et al $^{13}$ where leukaemia constituted the commonest malignancy causing PUO. In a study done by De Kleijn et al, $^{13}$ which was a prospective multicentric study of 167 cases of PUO, neoplasm constituted $12.6 \%$ of total cases. Haematological malignancies were $66.66 \%$ of total neoplastic cases. Hodgkin disease was the commonest neoplasm (35.7\%). In the study done by Knokaertet $\mathrm{al}^{14}$ and colleagues, $7 \%$ cases were malignancy as a cause of PUO. Haematological malignancy constituted 6 cases $(3 \%)$ and solid tumours constituted 8 cases (4\%). Among the haematological malignancies AML was the commonest, 3 cases (50\%). Multiple myeloma constituted only 1 case $(16.66 \%)$ and Hodgkin disease 2 cases (33.33\%). The results of these studies were similar to our study.
In our study, megaloblastic anaemia was the second most common cause $(16 \%)$ of pyrexia of unknown origin in adult. This was in concordance with study done by Davidson $\mathrm{S}$ et $\mathrm{al}^{15}$ where it occurred in $22 \%$ of patient, Davidson related the degree and frequency of fever to the severity of anaemia. According to McKee LC et $\mathrm{al}^{16}$ fever in megaloblastic anaemia is due to increased activity of megaloblastic marrow, and fever was present in about $40 \%$ of patients. Studies have shown that however the cause of pyrexia in megaloblastic anaemia is not exactly known but chance could be due to a defect in oxygenation to the regulatory centres of temperature in the brain secondary to anaemia due to vitamin B12 and folate deficiency. ${ }^{15,16,17,18}$ There was one other proposed mechanism for pyrexia in megaloblastic anaemia suggesting increased bone marrow activity to be responsible for the pyrexia but the exact mechanism is still not known.

In present study hypocellular marrow is seen in 04 cases (5\%). Various drugs, chemicals, toxins, infection, radiation or immune disorders were involved in aetiology of hypocellular marrow. Bone marrow examination alone was not sufficient to point out the cause of hypoplastic marrow. In patients with hypoplastic marrow, bacterial and fungal infections were common secondary to neutropenia. Haemophagocytosis was seen in 05 cases $(6 \%)$ in the present study. Viruses such as Herpes virus, Parvovirus-B19, CMV, EBV, and HIV are most commonly associated with haemophagocytosis. Although bacteria such as Salmonella, E.coli, Brucella, Legionella, and parasites such as Toxoplasma, Leishmania, and Malaria are also responsible for haemophagocytosis. ${ }^{19,20,21}$

Mirdha BR et $\mathrm{al}^{22}$ identified malaria in the bone marrow of 8 of 120 cases with PUO. Five cases were Plasmodium vivax and 3 cases were Plasmodium falciparum. In present study 04 cases of plasmodium vivax were diagnosed on bone marrow. The commonly used laboratory method for diagnosis of malaria in this part of world is microscopic examination of Romanowsky's stained thin and thick peripheral blood film. However, its sensitivity is directly proportional to the microscopic skill, screening time and staining. However diagnostic bone marrow examination is often performed when a patient with suspected infection has persistent fever. Malaria surveys based on microscopic examination of the blood film do not always detect chronic, low-grade infection due to either scanty parasitaemia or the patient's immunity. It is understood that till date complete consensus on routine diagnostic use of bone marrow for the diagnosis of malaria have not been achieved due to its inherent limitations. But, bone marrow examination still has a valuable place in the investigation of patients with suspected malaria. ${ }^{22}$

Reactive myeloid hyperplasia constituted 14 cases (18\%). This condition was non-specific morphological change of bone marrow in response to various inflammatory and infective conditions. No specific etiological agents were identified. Bone marrow responds to inflammation by accelerated release of cells from post mitotic reserve pool caused by IL-1 \& TNF and associated with an increase in numbers of more immature granulocytes. Severe bacterial infections lead to granulocytic hyperplasia with or without maturing cells. Toxic granules may be evident in the cytoplasm of the granulocytes. ${ }^{23}$ Bone marrow culture in association with bone marrow morphology would be more useful in suspected infectious aetiology. ${ }^{24}$ In our study, Leishmaniasis was detected in 03 cases (4\%). Study has been carried out showing the sensitivity of bone marrow 
being equivalent to splenic aspirates for diagnosing leishmaniasis. ${ }^{25}$

\section{CONCLUSIONS}

Bone marrow examination is an important investigation of PUO in arriving at an etiological diagnosis. The most frequent causes of pyrexia of unknown origin observed in children were acute lymphoblastic leukemia, megaloblastic anaemia and haemophagocytosis, whereas in adults, the main causes were malignancies, megaloblastic anaemia and reactive myeloid hyperplasia. This study sheds light on the current spectrum of diseases causing pyrexia of unknown origin in this region.

\section{REFERENCES}

[1] Petersdorf RG, Beeson PB. Fever of unexplained origin: report of 100 cases. Medicine (Baltimore) 1961;40(1):130.

[2] De Kleijn EM, Vandenbroucke JP, Van der Meer JW. Fever of unknown origin (FUO). I. A prospective multicenter study of 167 patients with FUO, using fixed epidemiologic entry criteria. The Netherlands FUO Study Group. Medicine (Baltimore) 1997;76(6):392-400.

[3] Zehnder JL. Bone marrow aspiration and biopsy: indications and technique. Website: http://www.uptodate.com/contents/bonemarrowaspiration-and-biopsy-indication-and-tec....] Retrieved on 15th April.

[4] Kumar S, Rau AR, Naik R, et al. Bone marrow biopsy in Non-Hodgkin's lymphoma: a morphological study. Ind J Pathol Microbiol 2009;52(3):332-8.

[5] Sipahi OR, Senol S, Arsu G, et al. Pooled analysis of 857 published adult fever of unknown origin cases in Turkey between 1990-2006. Med Scimonit 2007;13(7):CR31822.

[6] Kapoor OP. Value of bone marrow examination in patient having pyrexia of unknown origin. [http://www.bhj.org/journal/20024403.jul/cost 434.htm] Retrieved on 15th April 2011].

[7] Ben-Baruch S, Canaani J, Braunstein R, et al. Predictive parameters for a diagnostic bone marrow biopsy specimen in the workup of fever of unknown origin. Mayo Clin Proc 2012;87(2):136-42.

[8] Hot A, Jaisson I, Girard C, et al. Yield of bone marrow examination in diagnosing the source of fever of unknown origin. Arch Intern Med 2009;169(21):2018-23.
[9] Diebold J, Molina T, Camilleri-Broet S, et al. Bone marrow manifestations of infections and systemic diseases observed in bone marrow trephine biopsy review. Histopathology 2000;37(3):199-211.

[10] Arnow PM, Flaherty JP. Fever of unknown origin. Lancet 1997;350(9077):575-80.

[11] Root RK, Petersdorf RG. Fever of unknown origin. In: Root RK, Waldvogel F, Corey L, et al, eds. Clinical infectious diseases. A practical approach. New York: Oxford Medical Publications 1999: p. 459-69.

[12] McCarthy PL. Fever. Pediatrics 1999;11:49-55.

[13] Haq SA, Alam MN, Hossain SM, et al. A study of prolonged pyrexia in Dhaka. Bangladesh Med Res Counc Bull 1996;22(1):33-42.

[14] Knockaert DC, Vanneste LJ, Vanneste SB, et al. Fever of unknown origin in the 1980s. An update of the diagnostic spectrum. Arch Intern Med 1992;152(1):51-5.

[15] Davidson S. Clinical picture of pernicious anaemia prior to the introduction of liver therapy in 1926 and Edinburg subsequent to 1944. Br Med J 1957;1(5013):241-3.

[16] Mckee LC Jr. Fever in megaloblastic anemia. South Med J 1979;72(11):1423-4.

[17] Singanayagam A, Gange N, Singanayagam A, et al. Folate deficiency presenting as pyrexia: a case report. Cases Journal 2008;1(1):275.

[18] Jones RV, Payne RW. The occurrence of fever in anemia. Practitioner 1960;184:618-20.

[19] Shirono K, Tsuda H. Parvovirus B19-associated haemophagocytic syndrome in healthy adults. British J Haematol 1995;89(4):923-6.

[20] McKenna RW, Risdall RJ, Brunning RD. Virus associated haemophagocytic syndrome. Hum Pathol 1981;12(5):395-8.

[21] Janka G, Imashuku S, Elinder G, et al. Infection and malignancy-associated haemophagocytic syndromes. haematology Oncology Clinics of North America 1998;12(2):435-44.

[22] Mirdha BR, Samantray JC, Mishra B, et al. Bone marrow examination for identifying Malaria in fever of unknown origin. J Assoc Physicians India 1999;47(2):177-9.

[23] Bain BJ, Clark DM, Lampert IA. Bone marrow pathology: infection and reactive changes. $2^{\text {nd }}$ edn. Oxford: Blackwell Science 1996: p. 51-87.

[24] Jha A, Sarda R, Gupta A, et al. Bone marrow culture Vs blood culture in FUO. J Nepal Med Assoc 2009;48(174):135-8.

[25] Da Silva MRB, Stewart JM, Costa CHN. Sensitivity of bone marrow aspiration in diagnosis of visceral leishmaniasis. Am J Trop Med Hyg 2005;72(6):811-4. 